\title{
ON THE ENDOMORPHISM RING OF A SIMPLE MODULE OVER AN ENVELOPING ALGEBRA
}

\author{
DANIEL QUILLEN ${ }^{1}$
}

Let $U$ be an associative algebra with identity over a field $k$ and let $M$ be a simple $U$-module. If the cardinality of $k$ is greater than $\operatorname{dim}_{k} M$, then there is a rather simple argument (see [1]) showing that any element $\theta$ of $\operatorname{Hom}_{U}(M, M)$ is algebraic over $k$. Namely if $\theta$ is not algebraic, then $M$ is a nonzero vector space over $k(\theta)$, so $\operatorname{dim}_{k} M \geqq \operatorname{dim}_{k} k(\theta) \geqq$ card $k$, the last inequality coming from the fact that the elements $(\theta-\lambda)^{-1}$ of $k(\theta)$ are linearly independent. In this paper we prove by a completely different argument that the same conclusion holds for algebras $U$ having a filtration like a universal enveloping algebra but without any cardinality assumption on $k$. I am grateful to Victor Guillemin for motivating the problem.

TheOREM. Let $k$ be a field and let $U$ be an associative algebra with identity over $k$ endowed with an increasing filtration $F_{0} U \subset F_{1} U \subset \ldots$ by $k$-subspaces such that

(i) $1 \in F_{0} U, F_{p} U \cdot F_{q} U \subset F_{p+q} U, U=U F_{p} U$.

(ii) gr $U=\oplus F_{p} U / F_{p-1} U$ is a finitely generated commutative $k$ algebra.

If $M$ is a simple $U$-module, then every element of $\operatorname{Hom}_{U}(M, M)$ is algebraic over $k$.

Proof. The proof is based on the following result from algebraic geometry [3, SGA 60-61, Expose IV, Lemma 6.7].

Generic Flatness Lemma. If $A$ is a noetherian integral domain, $B$ is an $A$-algebra of finite type ( $A$ and $B$ are both commutative), and $N$ is a finitely generated $B$-module, then there is a nonzero element $f$ of $A$ such that $N_{f}$ is free over $A_{f}$.

Let $\theta \in \operatorname{Hom}_{U}(M, M)$; as $M$ is simple $\operatorname{Hom}_{U}(M, M)$ is a skew-field so the subring $k[\theta]$ generated by $\theta$ is an integral domain. Regard $M$ as a $k[\theta] \otimes_{k} U$ module in the obvious way, let $m_{0}$ be a nonzero element of $M$ so that $U m_{0}=M$, and filter $M$ by

$$
F_{p} M=k[\theta] \cdot F_{p} U \cdot m_{0} .
$$

Then $\mathrm{gr} M=\oplus F_{p} M / F_{p-1} M$ is a finitely generated module over

Received by the editors March 15, 1968.

${ }^{1}$ This research was supported in part by NSF GP-6959. 
$B=k[\theta] \otimes_{k}$ gr $U$ which in turn is a finitely generated commutative algebra over $A=k[\theta]$ by hypothesis (ii). By the generic flatness lemma there is a nonzero element $f$ of $k[\theta]$ such that $(\mathrm{gr} M)_{f}$ is free over $k[\theta]_{f}$. As localization is exact $(\operatorname{gr} M)_{f}=\operatorname{gr}\left(M_{f}\right)$ where $M_{f}$ is filtered by $F_{p}\left(M_{f}\right)=\left(F_{p} M\right)_{f}$; this filtration exhausts $M_{f}$ by hypothesis (i), so we conclude that $M_{f}$ is free over $k[\theta]_{f}$. But as $\operatorname{Hom}_{U}(M, M)$ is a skew-field $M$ is a vector space over the quotient field $k(\theta)$ of $k[\theta]$, so $M=M_{f}$ is a direct sum of copies of $k(\theta)$ as a module over $k[\theta]_{f}$. This means that $k[\theta]_{f} \simeq k(\theta)$ which can happen only if $\theta$ is algebraic over $k$.

Q.E.D.

The theorem applies to the universal enveloping algebra of a finite dimensional Lie algebra, hence the results of Dixmier [1] are valid without the assumption uncountability of $k$. In particular we have the following.

ThEOREM. Let $\mathrm{g}$ be a nilpotent Lie algebra of finite dimension over a field $k$ of characteristic zero. If $M$ is a simple $\mathrm{g}$-module, then the annihilator of $M$ is a maximal ideal in $U(\mathfrak{g})$.

Proof. If $I$ is the annihilator of $M$, then the center of $U(\mathfrak{g}) / I$ is isomorphic to a subring of $\operatorname{Hom}_{U(\mathfrak{g})}(M, M)$ and hence by the theorem is algebraic over $k$. This center is therefore a field so by Corollary 2.4 of [2], $I$ is maximal.

Q.E.D.

\section{BIBLIOGRAPHY}

1. J. Dixmier, Représentations irrèductibles des algèbres de Lie nilpotentes, An. Acad. Brasil. Ci. 35 (1963), 491-519.

2. Y. Nouazé and P. Gabriel, Ideaux premiers de l'algèbra enveloppante d'une algèbra de Lie nilpotente, J. Algebra 6 (1967), 77-99.

3. A. Grothendieck, Séminaire de géométrie algébrique, Notes, Inst. des Hautes Etudes Scientifiques, 1960-1961.

Massachusetts Institute of Technology 